\title{
Performance Evaluation of Reactive and Proactive Routing Protocols over MANET
}

\author{
NeerajBhargava,PhD \\ Associate Professor, \\ Dept. of Computer \\ Science, School of \\ Engineering \& System \\ ScienceS,MDS \\ University, Ajmer, India
}

\author{
Ritu Bhargava,Ph.D \\ Dept. of MCA, \\ Govt. Women \\ Engineering College, \\ Ajmer, India
}

\author{
Manish Mathuria \\ Dept. of C. E. \& I. T. \\ Govt. Engineering \\ College, \\ Ajmer, India
}

\author{
Anchal Kumawat \\ Dept. of Computer \\ Science, School of \\ Engineering \& System \\ Sciences, \\ MDS University, \\ Ajmer, India
}

\begin{abstract}
Mobile devices are the fundamental requirement of communication network where user wants to freely move without breaking the signal. The Mobile devices today are capable enough to communicate independently. A Mobile Ad Hoc Network (MANET) is a group of wireless mobile nodes which can communicate without any pre-existing infrastructure by creating dynamic network. This paper discuss Mobile Ad-Hoc Network (MANET) environment with the varying number of nodes. The research presents the three reactive routing protocols i.e. Dynamic Source Routing (DSR), Destination Sequence Distance Vector and Ad hoc On Demand Distance Vector (AODV) with their results to the MANET in terms of packet delivery ratio, delay, routing load and throughput. Simulation is carried out by NS2 (Network Simulation). The experiment results reflect that AODV performance it better than DSR DSDV.
\end{abstract}

\section{Keywords}

MANET, NS2, DSDV, DSR, AODV, PDR, NRL, Routing protocols.

\section{INTRODUCTION}

The term 'MANET' stands for Mobile Ad hoc Network. It is a special collection of independent wireless mobile nodes that can communicate each other via radio waves. The advantage of MANET is, one can connect any wireless physical device like computer, PDA, Laptops and our cell phones. Infrastructure of MANET is not fixed. One can establish it, at any time. The major advantage of MANET is, one can create infrastructure at real time and destroy it after completion work. Military and disaster areas are the application example of MANET. MANET provides each node to freely move in any arbitrary fashion, independently. The topology changes frequent and Multi- hop wireless links. Data routed via the intermediate nodes, so each mobile node can be react either as endpoint or as a router to forward the packets to the next node. In contrast to fixed infrastructure networks, MANET requires some fundamental changes to network routing protocols [1] [2].

The Heading-2 defines the Routing protocols of MANET to discover the route between nodes. And Heading-3, defines Performance Metrics (packet delivery ratio, delay, routing load and throughput) in terms of evaluating the performance of three routing protocols. The Heading- 4 discusses about the analysis of simulation results.

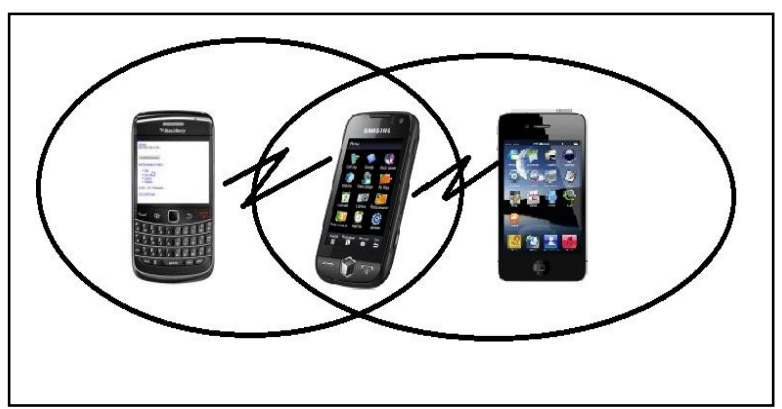

Fig 1: A Simple Mobile Ad hoc Network

\section{ROUTING PROTOCOLS IN MOBILE} AD HOC NETWORK

The routing protocols in Mobile Ad-hoc Network can be broadly divided into three categories:

\subsection{Proactive or Table-driven routing protocols}

Pro-active routing protocol is also known as Table-driven routing protocol because routing protocol requires each node to maintain routing information to the every other node in the network which should be consistent up-to-date routing information. On-demand routing protocols are mainly designed for the purpose is to reduce the overheads in table-driven protocols by maintaining information for active routes and each node to maintain one or more tables to store routing information and responds to changes in network topology by propagating updates throughout the network.

\subsubsection{Destination-Sequenced Distance-Vector Routing (DSDV)}

The Destination-Sequenced Distance-Vector Routing protocol (DSDV) is a Proactive Routing Protocol. This Proactive routing protocol algorithm is based on the classical Bellman-Ford routing mechanism.

Each node stores information about all destinations and number of hops to each destination in table. Each entry is marked with a unique sequence number. DSDV uses two mechanisms such as full dump and incremental update to reduce network traffic generated by rout updates. 
A full dump sends the full routing table to the neighbors whereas in case of incremental update, only those entries are sent from the routing table that has a metric change since the last update and must be fit in a packet. In Incremental update those entries may be included whose sequence number has changed if space is available.

There may be two cases are possible about incremental update and full dump such as -

1. If the network is relatively stable, then incremental updates are sent to avoid extra traffic and full dump relatively refer as infrequent.

2. If the network is changing fast, then incremental packets can grow big and full dumps will refer as more frequent.

DSDV solves the problem of routing loops and count to infinity by associating each route entry with a unique sequence number which indicates that the packet is fresh means same packet is not transmitted again. In DSDV, a sequence number is linked to a destination node, and usually is originated by that node refer as owner. The list which maintained all information about route is called routing table. The routing table consist important information such as IP address of all destinations, hop IP address (which prefers as next), total number of hops to reach the destination, sequence number assigned by the destination node and last it consist the install time.

Updating sends in a proper form of-

<Destination IP address, Destination sequence number, Hop count >

Neighbor nodes utilize receiving updates to compute the routing table entries. If there are any fluctuations or breaks the link between two nodes which shown as in figure 2 "resolving failed links" due to periodic updates then Routing will updates for a given destination. It broadcast to all neighbors and then link will be established to transfer the packet. To prevent a node from announcing a routing path change for a given destination DSDV requires node to wait a settling time before announcing a new route with higher metric for a destination [3].

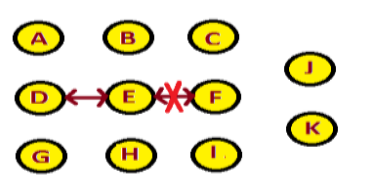

Fig 2 (a) Link from $E$ to F Breaks

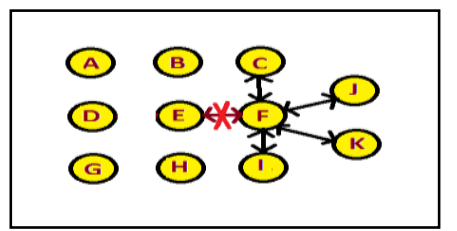

Fig 2(b) F broadcast route request to its neighbor

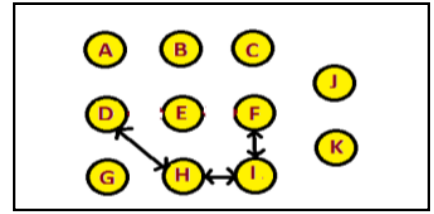

Fig 2 (c) Links established

\subsection{Reactive or On-demand routing protocols}

A Reactive routing protocol is also known as On-Demand Routing Protocol. On-Demand is the sense that discovers the routes on demand. It reduces the overheads of Proactive routing protocol such as maintaining the route table or reduces the signaling overhead.

A proactive routing is on-demand routing. This type of routing mainly used to create the route, only when the source node wants to send the data to destination. If a source node does not find the route to the destination then it initiates route discovery process within the network. Once a route has found or established, it is maintained by the form of route maintenance procedure.

Route maintenance check for the destination that is inaccessible along the path from the source or until the route is no longer desired then maintains the route or sends this break information to every other intermediate node for recovery.

Reactive routing protocol consist one drawback of route acquisition latency. Route acquisition latency refers that when corresponding entry is not found then route discovery mechanism takes place due to this it takes a very large amount of time, and for that time the packet should wait till updating of the table [4] [5] [6].

\subsubsection{Dynamic Source Routing (DSR)}

Dynamic Source Routing is a Reactive routing protocol. The main feature of DSR is to use the source routing. DSR makes very aggressive use of source routing and route caching. It means sender knows the complete route to the destination. These routes are stored in a route cache. When a source node wants to send the data to a destination and it does not know the route then it uses a route discovery process to determine a route. Route discovery works by flooding the network with route request (RREQ) packets. Each node which receives an RREQ it rebroadcasts to the next intermediate nodes unless it reach to the destination. Such a node replies to the RREQ with a route reply (RREP) packet that is routed back to the original source.

For the Route Discovery Route Request and Route Reply both are shown as in figure 3 (a) and (b). RREQ and RREP packets are also source routed. RREQ (S creates route request from $S$ to D (destination node) and RREP (from D Destination to source S). Now another Mechanism of DSR is Route Maintenance. In case of Route Maintenance recover the failure links. If any link on a source route is broken, the source node is notified using a route error (RERR) packet. The source removes that route from cache which consist error then, a new route discovery process must be initiated by the source if this route is still needed which shown as in figure 4 (a) and (b) [7] [8] [9]. 


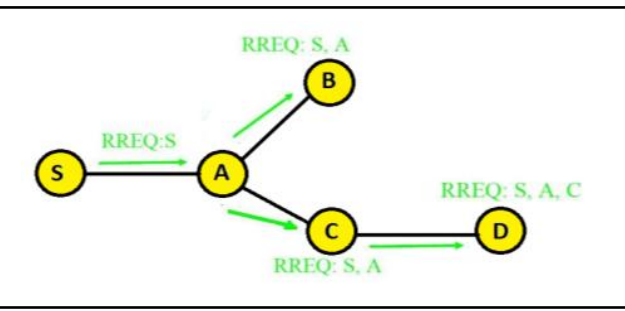

Fig. 3 (a) For Route Discovery initiates Route Request (RREQ) in DSR

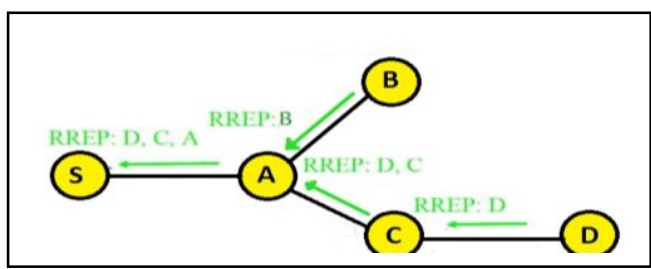

Fig. 3 (b) For Route Discovery Route Reply (RREP) in DSR

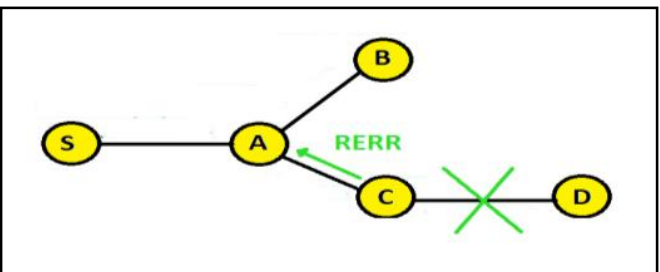

Fig. 4 (a) For Route Maintenance Route Error (RERR) in DSR

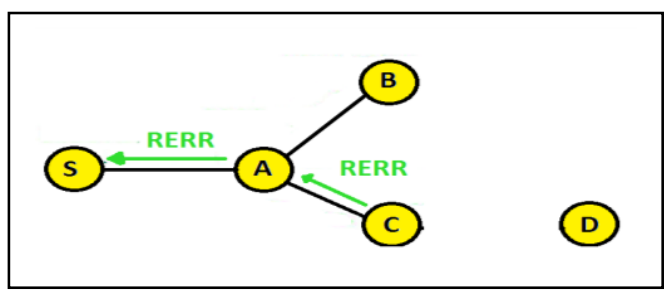

Fig. 4 (b) For Route Maintenance broadcast Route Error (RERR) in DSR

\subsubsection{Ad hoc On-Demand Distance Vector Routing (AODV)}

The Ad-hoc On-Demand Distance Vector (AODV) routing protocol is a Reactive routing protocol which build on the DSDV algorithm. AODV is an just an advancement of DSDV because it minimizes the number of required broadcasts by creating routes on-demand basis, in case of DSDV maintaining a complete list of routes.

Main features of AODV consist such as: each route has a lifetime after which the route expires if it is not used and a route is maintained only when it is used and old and expired routes are never used and AODV maintains only one route between a source-destination pair. AODV does not retransmit data packets that are lost. The overhead packets in AODV are due to RREQ (Route Request), RREP (Route Reply) and RERR (Route Error) messages.
AODV needs less number of overhead packets as compared to proactive protocol such as DSDV.

With increased mobility, the number of overhead packets will also increase due to this it give rise to frequent link breaks and route discovery. AODV uses traditional routing tables, one entry for per destination. AODV relies on routing table entries to propagate an RREP back to the source and, subsequently, to route data packets to the destination. AODV uses sequence numbers maintained at each destination to determine freshness of routing information and to prevent routing loops. All routing packets carry these sequence numbers [10] [11].

\subsection{Hybrid routing protocols}

Hybrid routing protocol is a combination of both Reactive and Proactive routing protocol. Firstly it performs proactive after that it performs reactive only if route does not exist in routing table so performs route discovery. For this we need to use reactive routing protocol.

\section{Performance Metrics}

The main goal of this research paper is to evaluate the performance of DSR, DSDV and AODV routing protocols using following metrics:

\subsection{Packet Delivery Ratio}

The Packet delivery ratio is the ratio of the number of delivered data packet to the destination.

$\mathrm{PDR}=\sum$ No. of packet receive $/ \sum$ No. of packet send

\subsection{End -to-End Delay}

End-to-end Delay is referred as the average time taken by a data packet to arrive in the destination. It also includes the delay caused by route discovery process and the queue in data packet transmission. Only the data packets that successfully delivered to destinations that counted.

End-to-End Delay $=\sum$ (arrive time - send time) $/ \sum$ No. of connections

\subsection{Normalized Routing Load}

The number of routing packets transmitted per data packet delivered at the destination. Each hop-wise transmission of a routing packet is counted as one transmission.

\subsection{Throughput}

Throughput refers that number of jobs completed in the given unit time. Throughput of the routing protocol means that how many packets are received in particular time interval. The unit of throughput measure in Kilobits per second (Kbps). 


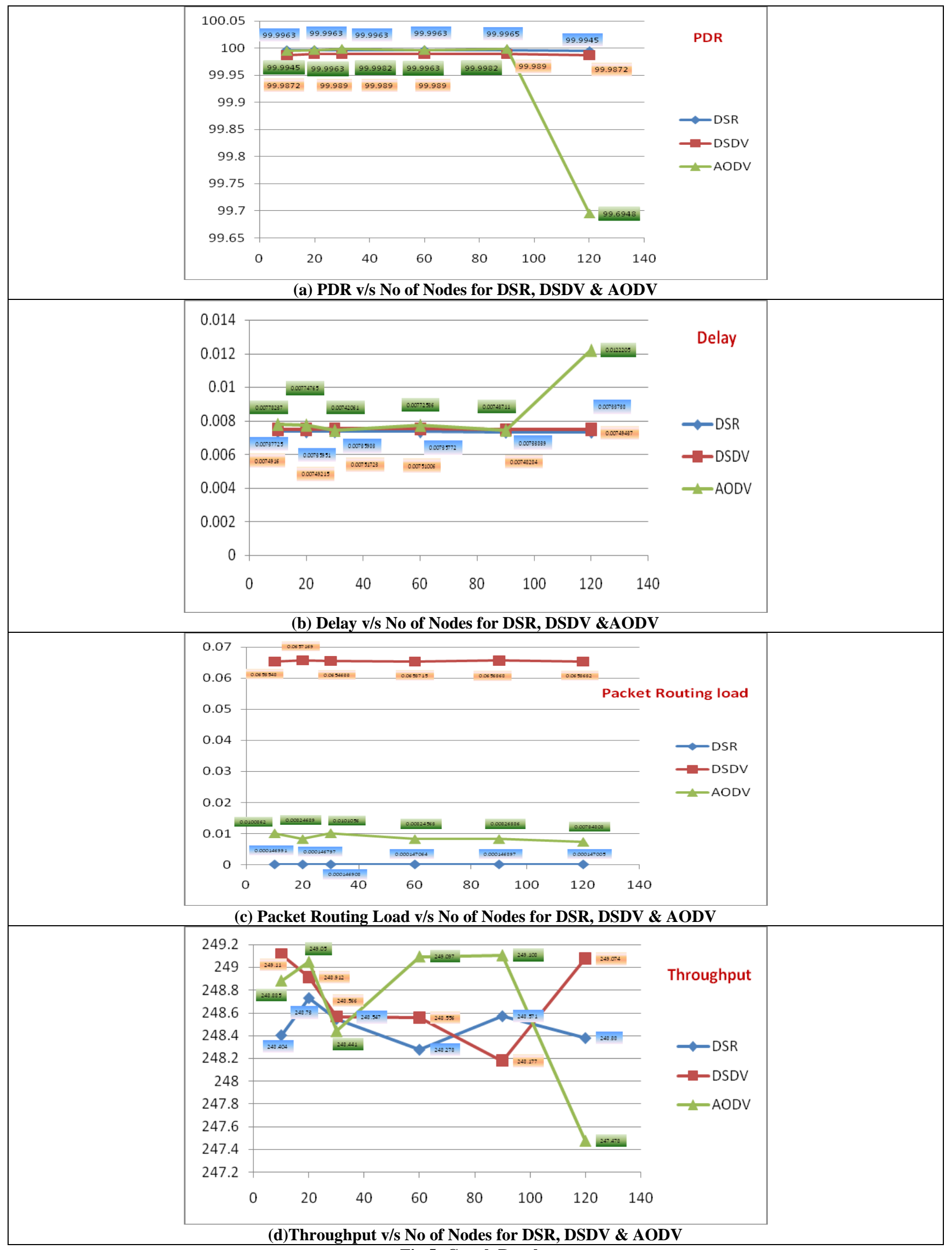

Fig 5: Graph Results 


\section{Simulation}

The simulations are carried out using Network Simulator-2 (Ns-2.35). Constant bit rate (CBR) traffic was used in the simulation. Simulation was done according to the number of nodes which vary from up to $10,20,30,60,90$ and 120 .

One need scenario file or traffic generation file for evaluating the performance of DSR, DSDV \& AODV protocol [12].

Traffic generation is achieve by running the CBR generation tcl file consist some parameters. The following line writes in terminal such as:

ns cbrgen.tcl

ns cbrgen.tcl -type cbr -nn 10 -seed $1.0-$ mc 8 -rate $4.0>$ cbr-10-test.

Whereas -

- type - refer as traffic /connection type

- $\quad$ nn-number of nodes

- $\quad$ seed - seed for generating random number it is used to generate the random starting time of the traffic.

- $\quad \mathrm{mc}-$ max. Number of connections

- rate - packet rate $=1$ / packet interval

The our main tcl-script will run, which evaluate the performance metrics -

ns wirelesseg.tcl AODV cbr-10-test scen-1-test ns wirelesseg.tcl DSR cbr-10-test scen-1-test ns wirelesseg.tcl DSDV cbr-10-test scen-1-test Then simulation result will be displayed on terminal. The following table deals with the simulation Parameters which is used during the simulation [13] [14] [15].

TABLE I

SIMULATION PARAMETER

\begin{tabular}{|l|l|}
\hline Parameter & Value \\
\hline Simulator & NS-2.35 \\
\hline Simulator Area & $500 \mathrm{mX} 500 \mathrm{~m}$ \\
\hline No. of Mobile & $10,20,30,60$, \\
\hline Pause Time & $80 \mathrm{sec}$ \\
\hline Packet Size & 512 \\
\hline Routing Protocols & DSR,DSDV\&AOD \\
\hline Traffic Sources & CBR \\
\hline Simulation Time & 900 Sec. \\
\hline
\end{tabular}

\subsection{Packet Delivery Ratio (PDR)}

Figure 5 shows that the $\mathrm{P}$ acket delivery ratio for reactive protocols. AODV and DSR were better than the proactive protocol DSDV according to the given table II results. From table II of PDR analysis, one can easily get the result i.e. DSDV Packet delivery ratio is smaller than DSR and AODV. In the given table II PDR analysis for AODV is varies when the number of nodes varies.
TABLE II

PDR ANALYSIS OF DSR DSDV AND AODY

\begin{tabular}{|l|l|l|l|}
\hline No. of Nodes & DSR & DSDV & AODV \\
\hline 10 & 99.9963 & 99.9872 & 99.9945 \\
\hline 20 & 99.9963 & 99.989 & 99.9963 \\
\hline 30 & 99.9963 & 99.989 & 99.9982 \\
\hline 60 & 99.9963 & 99.989 & 99.9963 \\
\hline 90 & 99.9965 & 99.989 & 99.9982 \\
\hline 120 & 99.9945 & 99.9872 & 99.6948 \\
\hline
\end{tabular}

\subsection{End to End Delay}

It is clear from the figure 6 that the lower value of end to end delay means the better performance of the protocol. Here, the DSR consist the better performance as comparisons of other two protocols i.e. DSDV and AODV which is shown as in Table III. From the given table "Delay Analysis of DSR DSDV and AODV" and the graph, one can easily get the delay in AODV is highest among DSR and DSDV. But the values of DSDV are slightly different from DSR so that the result proves the DSR is better than both Reactive (AODV) and Proactive (DSDV) Protocols over varying nodes.

TABLE III

DELAY ANALYSIS OF DSR DSDV AND AODV

\begin{tabular}{|l|l|l|l|}
\hline No. of Nodes & DSR & DSDV & AODV \\
\hline 10 & 0.00737725 & 0.0074916 & 0.00778237 \\
\hline 20 & 0.00735951 & 0.00749215 & 0.00774765 \\
\hline 30 & 0.00735983 & 0.00751723 & 0.00742061 \\
\hline 60 & 0.00735772 & 0.00751006 & 0.00772536 \\
\hline 90 & 0.00733839 & 0.00748284 & 0.00743711 \\
\hline 120 & 0.00733738 & 0.00749437 & 0.0122205 \\
\hline
\end{tabular}

4.3 Normalized Routing Load (NRL)

As shown as in figure 7, normalized routing load for DSR is smaller as compare with DSDV and AODV routing protocol. The Routing load for AODV was better than the DSDV protocol because AODV values are smaller then DSDV. Here DSR and AODV perform better result in normalized packet routing Load and DSDV performs higher load as we can see in figure 7 . 
TABLE IV

NRL ANALYSIS OF DSR DSDV AND AODV

\begin{tabular}{|l|l|l|l|}
\hline No. of Nodes & DSR & DSDV & AODV \\
\hline 10 & 0.000146991 & 0.0653548 & 0.0100862 \\
\hline 20 & 0.000146797 & 0.0657169 & 0.00824689 \\
\hline 30 & 0.000146908 & 0.0654633 & 0.0101056 \\
\hline 60 & 0.000147064 & 0.0653715 & 0.00824563 \\
\hline 90 & 0.000146897 & 0.0656368 & 0.00826386 \\
\hline 120 & 0.000147005 & 0.0653632 & 0.00734808 \\
\hline
\end{tabular}

\subsection{Throughput}

Figure 8 evaluate the performance of three protocols in respect to throughput analysis. So, it is clear from to the table statistics that lowest throughput received from the proactive routing protocols. According to results AODV react better in case of throughput analysis.

TABLE V

THROUGHPUT OF DSR, DSDV AND AODV

\begin{tabular}{|l|l|l|l|}
\hline No. of Nodes & DSR & DSDV & AODV \\
\hline 10 & 248.404 & 249.11 & 248.885 \\
\hline 20 & 248.73 & 248.912 & 249.05 \\
\hline 30 & 248.547 & 248.566 & 248.441 \\
\hline 60 & 248.278 & 248.556 & 249.097 \\
\hline 90 & 248.571 & 248.177 & 249.108 \\
\hline 120 & 248.38 & 249.074 & 247.478 \\
\hline
\end{tabular}

\section{CONCLUSION}

In research paper, the evaluation is done by calculating the performance reactive (DSR and AODV) and proactive (DSDV) routing protocols in the terms of four performance metrics i.e. packet delivery ratio, end-to-end delay, normalized routing load and throughput. The results showed that the performance of the two reactive protocols (DSR and AODV) was better than proactive protocol (DSDV). The overall performance of DSR was better than the other two protocols except in the case of throughput. DSR consist lower throughput value which mainly due to caching and lack of mechanisms to expire stale routes. The performance of AODV was comparable to DSR in case of packet delivery ratio and throughput; it was better in case of end to end delay and inferior in case of normalized routing load.

\section{REFERENCES}

[1] Elizabeth M. Royer, Santa Barbara, Chai-Keong Toh, "A Review of Current Routing Protocols for Ad Hoc Mobile Wireless Networks" 1070-9916/99/ IEEE Personal Communications, April 1999.

[2] Mehran Abolhasan a, Tadeusz Wysocki a, Eryk Dutkiewicz,"A review of routing protocols for mobile ad hoc networks", Motorola Australia Research Centre, 12 Lord St., Botany,NSW 2525, Australia. 4 June 2003.

[3] Perkins, C.E.; Bhagwat, P.; : Highly dynamic destination-sequenced distance vector routing (DSDV) for mobile computers, Proceedings of ACM SIGCOMM 94, 1994, pp. 34-244.

[4] Hu, Y.C., Johnson, D.B., Perrig, A.: Ariadne: A secure on-demand routing protocol for ad hoc networks. Proc. 8th Ann. Intl Conf. Mobile Computing and Networking (MobiCom 2002), ACM Press pp. 12-23 (September 2002).

[5] Study of routing protocols in mobile ad-hoc networks, vol. 3, no. 4, April 20112622 in “. Introduction".

[6] Simulation Based Performance Evaluation of MANET Routing Protocols in International Journal of Computer Science and Management Research Vol. 1, issue 4 November 2012, ISSN 2278-733X.

[7] Fall, K., Varadhan, K.: Ns-2, the ns manual (formally known as ns documentation) available at http: //www. isi.edu/nsnam/ns/doc.

[8] Rendong Bai and Mukesh Singhal," DOA: DSR over AODV Routing for Mobile Ad Hoc Networks" IEEE Trans on Mobile Computing, vo1. 5, no.IO, 2006.

[9] D.B. Johnson and D.A. Maltz, "Dynamic Source Routing in Ad Hoc Wireless Networks," Mobile Computing, vol. 353. Kluwer Academic, 1996.

[10] Johnson, D.B; Maltz, D.A; Hu, Y.C.; : The Dynamic Source Routing Protocol for Mobile Ad Hoc Networks (DSR), IETF Draft, April 2003, work in progress.

[11] C. E. Perkins and E. M. Royer. The Ad hoc On-Demand Distance Vector Protocol. In C. E. Perkins, editor, Ad hoc Networking, pp. 173-219. Addison-Wesley, 2000.

[12] Ian D. Chakeres and Elizabeth M. Belding-Royer. AODV Routing Protocol Implementation Design.

[13] Per Johansson, Tony Larsson, Nicklas Hedman, and Bartosz Mielczarek. Routing protocols for mobile ad-hoc networks - a comparative performance analysis. In proceedings of the 5th International Conference on Mobile Computing and Networking (ACM MOBICOM '99), August 1999, pp. 195- 206.

[14] Misra ,R.; Manda,C.R.; : Performance Comparison of AODV/DSR On-Demand Routing Protocols for Ad Hoc Networks in Constrained Situation, IEEE ICPWC 2005.

[15] A. Boukerche, "Performance Evaluation of Routing Protocols for Ad Hoc Wireless Networks "Mobile Networks and Applications", Vol. 9, pp. 333-342, Kluwer Academic Publishers. Manufactured in The Netherlands, 2004. 